\title{
Celebrate the renewal of Analytical Sciences
}

\author{
Takeshi Hasegawa $^{1}$
}

Published online: 19 February 2022

(c) The Author(s), under exclusive licence to The Japan Society for Analytical Chemistry 2022

We, the editorial team, are very happy to publish the present special issue that involves review papers written by the executive associate editors (EAEs) of Analytical Sciences. This issue is, in addition, a very special issue with another meaning that the journal has first been published by Springer Nature. Here, the Japan Society for Analytical Chemistry (JSAC) and Springer Nature are proud of starting cooperative publication of Analytical Sciences from January 2022.

Analytical Sciences was launched by JSAC in 1985, and it has long been published by JSAC alone for many years thus far. In recent years, very many scientific manuscripts of analytical chemistry, physics and mathematics are submitted in an increasing manner, and the publication media becomes short for the active community. To fulfill the demand, a new publication medium in Asia would be needed as the third core in addition to EU and US, and JSAC was decided to start collaboration with Springer Nature to re-launch Analytical Sciences to make it more powerful. The current issue is the memorable first issue of the renewed journal.
The first issue is, fortunately, colored by nine great review papers written by very active scientists working as EAEs covering electrochemical sensor, pharmaceutical analysis, Raman spectroscopy, artificial intelligence technology on neural networks, chemical sensor using carbon nanomaterials, and paper-based analytical device. This range straightforwardly implies that Analytical Sciences receives a wide range of "analytical sciences," which does not repel manuscripts out of analytical chemistry in a narrow sense.

We welcome scientific manuscripts in analytical sciences in a wide sense, and we are trying to review them in an unbiased manner as possible. If you have good experimental or theoretical results with an original idea, please consider Analytical Sciences for your future success. Not only methodological novelty, but application novelty in a wide area is also welcome.
Takeshi Hasegawa

htakeshi@scl.kyoto-u.ac.jp

1 Institute for Chemical Research, Kyoto University, Kyoto, Japan 